\title{
Postoperative Myoclonus After Epilepsy Surgery: A Case Report
}

\author{
Maryam Khaleghi Ghadiria , Christoph Kellinghaus ${ }^{\mathrm{b}}$, Mohsen Aghaee Hakak ${ }^{\mathrm{c}}$, Ali Goji ${ }^{\mathrm{d}}$ e, f, \\ Walter Stummer ${ }^{\mathrm{a}}$
}

\begin{abstract}
Accurate diagnosis of myoclonus after epilepsy surgery is important to determine the surgical outcome in patients with intractable epilepsy. Emerged bilateral myoclonus in a 53-year-old man after surgical resection for treatment of traumatic refractory epilepsy is reported. An electroencephalography correlate of the myoclonic jerks with cortical potentials was not observed. Treatment with clonazepam significantly reduced the myoclonic twitching. The possible mechanism and differential diagnosis between non-epileptic and epileptic myoclonus following brain surgery are discussed. To avoid misdiagnosis and ensure appropriate treatment, the origin of jerking mimicking epileptic myoclonus after brain surgeries needs to be carefully investigated.
\end{abstract}

Keywords: Intractable epilepsy; Neuroimaging; Brain; Neocortex; Tremor

\section{Introduction}

Myoclonus is a brief, shock-like, involuntary jerking of a muscle or a group of muscles caused by sudden muscle contractions or relaxation and may begin in one muscle and spread to muscles in other areas of the body. Four major types of myoclonus are classified into physiologic, essential, secondary (symptomatic), and epileptic [1]. Epileptic myoclonus, the second most common type of myoclonus, is referred to the presence of myoclonus associated with ictal spike-and-

Manuscript accepted for publication March 24, 2016

aDepartment of Neurosurgery, Westfalische Wilhelms-Universitat Munster, Germany

bDepartment of Neurology, Klinikum Osnabruck, Osnabruck, Germany

${ }^{\mathrm{c}}$ Razavi Neuroscience Center, Razavi Hospital, Mashhad, Iran Neurovascular Research, Iran

dEpilepsy Research Center, Department of Neurology, Westfalische WilhelmsUniversitat Munster, Germany

eShefa Neuroscience Research Center, Khatam-Alanbia Hospital, Tehran, Iran ${ }^{f}$ Corresponding Author: Ali Gorji, Epilepsy Research Center, Universitat Munster, Robert-Koch-Straße 27a, D-48149 Munster, Germany.

Email: gorjial@uni-muenster.de

doi: http://dx.doi.org/10.14740/jmc2471w wave electroencephalography (EEG) discharges, time locked with and preceding muscle twitching [2]. Although epileptic myoclonus is associated with bilaterally synchronized epileptiform discharges, the myoclonus itself may exhibit as generalized, segmental or focal muscle jerks. Focal myoclonus can be caused by trauma, infection, tumors, vascular diseases, and neuroinflammation $[3,4]$. In addition, focal myoclonic seizure has been reported to occur during surgical procedures due to administration of anesthetic agents [5] or after brain resection due to operative failure in terms of seizure control, coexisting generalized epilepsy or a complication of brain resection [6-8]. Occurrence of myoclonus following a left temporal lobectomy for chronic encephalitis [6] as well as after standard anterior temporal lobectomy for medically intractable temporal lobe epilepsy has been reported [8]. Few days or weeks after the operation, the patients developed restlessness of fingers and hands accompanied by atypical $2 \mathrm{~Hz}$ spike-wave from a temporal focus [6] or brief bilateral jerks occurring weekly and always with clear consciousness [8]. Here, we present a patient with traumatic intractable epilepsy who developed myoclonus after brain surgery mimicking epileptic myoclonus, and discuss the importance of understanding of the origin of myoclonus in the management of the patients.

\section{Case Report}

A 53-year-old right-handed man who suffered from epileptic seizures starting 6 months after a severe penetrating head injury in war 30 years ago with remaining metal fragments in the right frontal lobe was referred to the Neurosurgery Department of Munster University. Informed consent was obtained from patient. Seizures consisted in clonic jerks of the left hand with preserved consciousness that tended to generalize convulsion. Treatment with almost all known antiepileptic drugs in the past had failed to produce any significant therapeutic effect. On referral, he was on maximal doses of levetiracetam, lacosamide, valproic acid, and phenytoin and suffered from a mild-to-moderate brachiofacial sensory-motor hemiparesis. Patient underwent long-term video EEG monitoring. Clinically, the patient started having jerky movements of the left hand, but remained responsive. After 5 - $10 \mathrm{~s}$, the jerks became more violent. In all but one seizure, generalization followed with right arm tonic extension, left arm tonic flexion, and subtle irregular jerking of the tonic arms. Face and trunk 


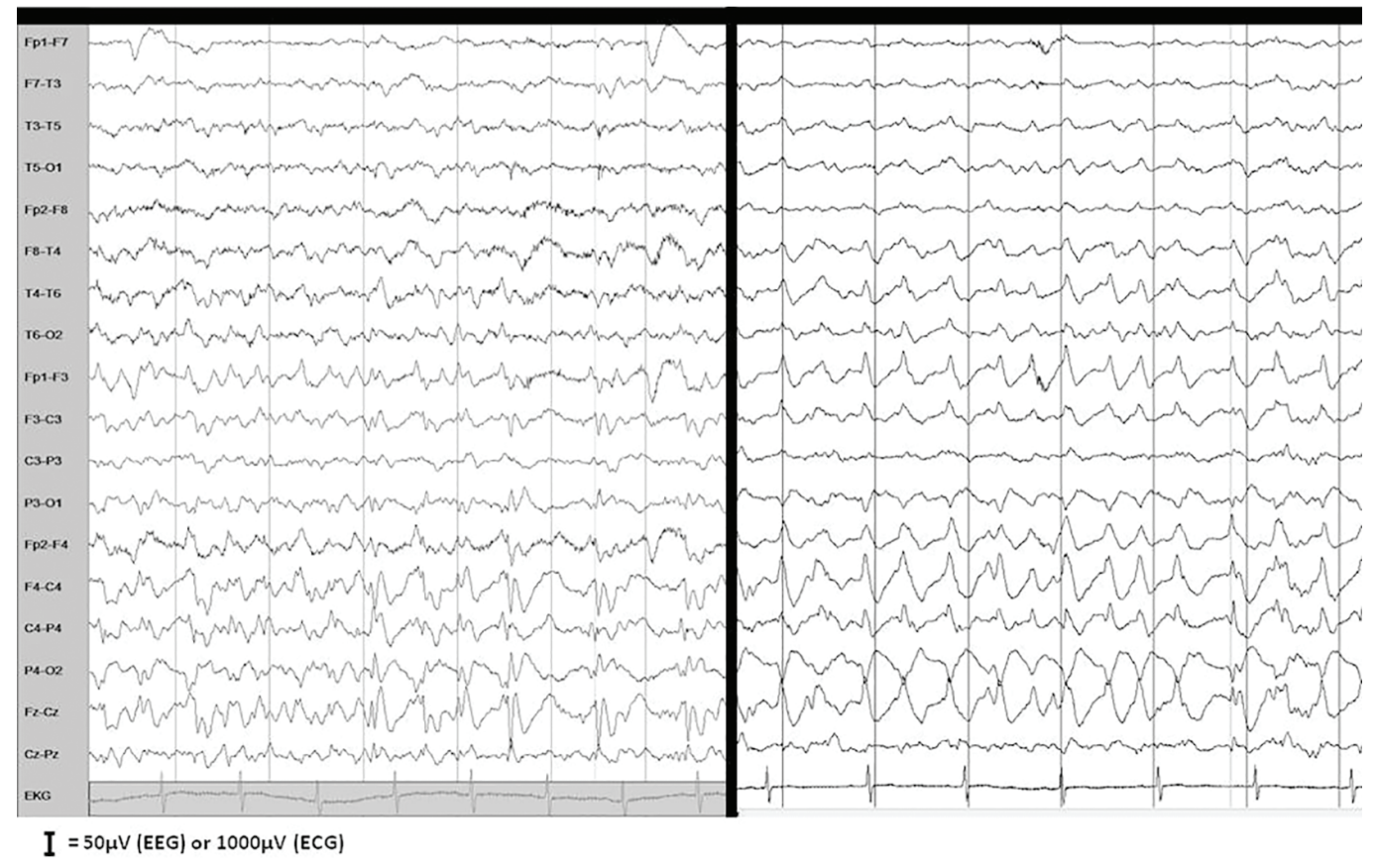

Figure 1. Interictal EEG (before vertical black line): spikes with phase reversal at C4/P4. Ictal EEG (after vertical black line): rhythmic activity with broad field, maximum at C4/P4. Filter settings: low frequency filter $1 \mathrm{~Hz}$, high frequency filter $70 \mathrm{~Hz}$, notch filter $50 \mathrm{~Hz}$ applied.

were involved bilaterally. At the end of some seizures, while still jerking, the patient seemed responsive. Rhythmic spiking/alpha activities were starting at right frontal lobe and then spread with rhythmic spiking bilaterally, predominantly to right frontocentral area. Seizure duration was 30 - 50 s. Interictally, there were frequent sharp waves with a right frontal/ frontopolar maximum that occurred at times in runs over 5 - 6 s. In addition, there was intermittent theta-delta-slowing in the right frontal region (Fig. 1). CT scan of the brain revealed a large hypodense defect zone in the right frontal lobe stretching from right frontobasal to right frontopolar regions, involving the anterior insula and the right frontal operculum, with two metal fragments located in the defect zone. The right lateral ventricle is enlarged and drawn to the outward, most likely communicating with the cerebrospinal fluid space of the defect zone. Large bone defect was observed from frontotemporal to frontopolar/frontocentral (Fig. 2a).

The patient underwent brain operation. Due to respiratory difficulties and bronchospasm of the patient produced by chemical warfare agent damages, intraoperative brain mapping by awake brain surgery was not possible. Initial identification of the central sulcus and motor strip was done by neuronavigation. A subdural strip of six electrodes was placed across the presumed central sulcus and using somatosensory evoked potential phase reversal method, the exact location of the central sulcus was identified. Electrocorticographic (ECoG) recordings were performed to identify and localize the extent of the cortex that generated epileptic seizures (epileptogenic zones) [9]. Tissue removal was carried out by subpial resection technique. Following removal of the indurated tissues in the frontal lobe, ECoG was used to detect epileptic activity remained at the resection margins and further removal of these centers was performed by microsurgery. Brain CT scan performed after operation showed features of the frontal lobe resection and removal of metal fragments (Fig. 2b). The patient did not suffer any apparent episode of hypoxic during surgery and there was no evidence of any infection after surgery. Preoperative seizures were abolished by surgical resection and he was seizure free since surgery after 4 years of follow-up. The day after resection, the patient had no significant new neurological deficit. However, 3 days after the operation, the patient developed continuous bilateral jerks, most prominently in the left side, occurring always with clear consciousness and became more violent with emotional stress and sensory stimuli. EEG recordings were performed to rule out occurrence of myoclonic status epilepticus [10]. Although EEG showed a few scattered bilateral frontal spikes, an EEG correlate of the myoclonic jerk with cortical potentials was not observed. Treatment with clonazepam given by oral route in doses of $0.5 \mathrm{mg}$ two times daily for a period of 1 week significantly reduced the myoclonic twitching. Currently, 4 years after operation and by receiving a daily dose of $0.5 \mathrm{mg}$ clonazepam, he suffered no more myoclonic jerks, while remaining seizure free.

\section{Discussion}

Postoperative myoclonus, which is not associated with EEG evidence of seizure activity, is a well-known side effect of general anesthesia during surgical procedures, especially induced by opiate derivatives [11]. Postoperative paraplegia with spinal myoclonus possibly caused by epidural anesthesia was also re- 

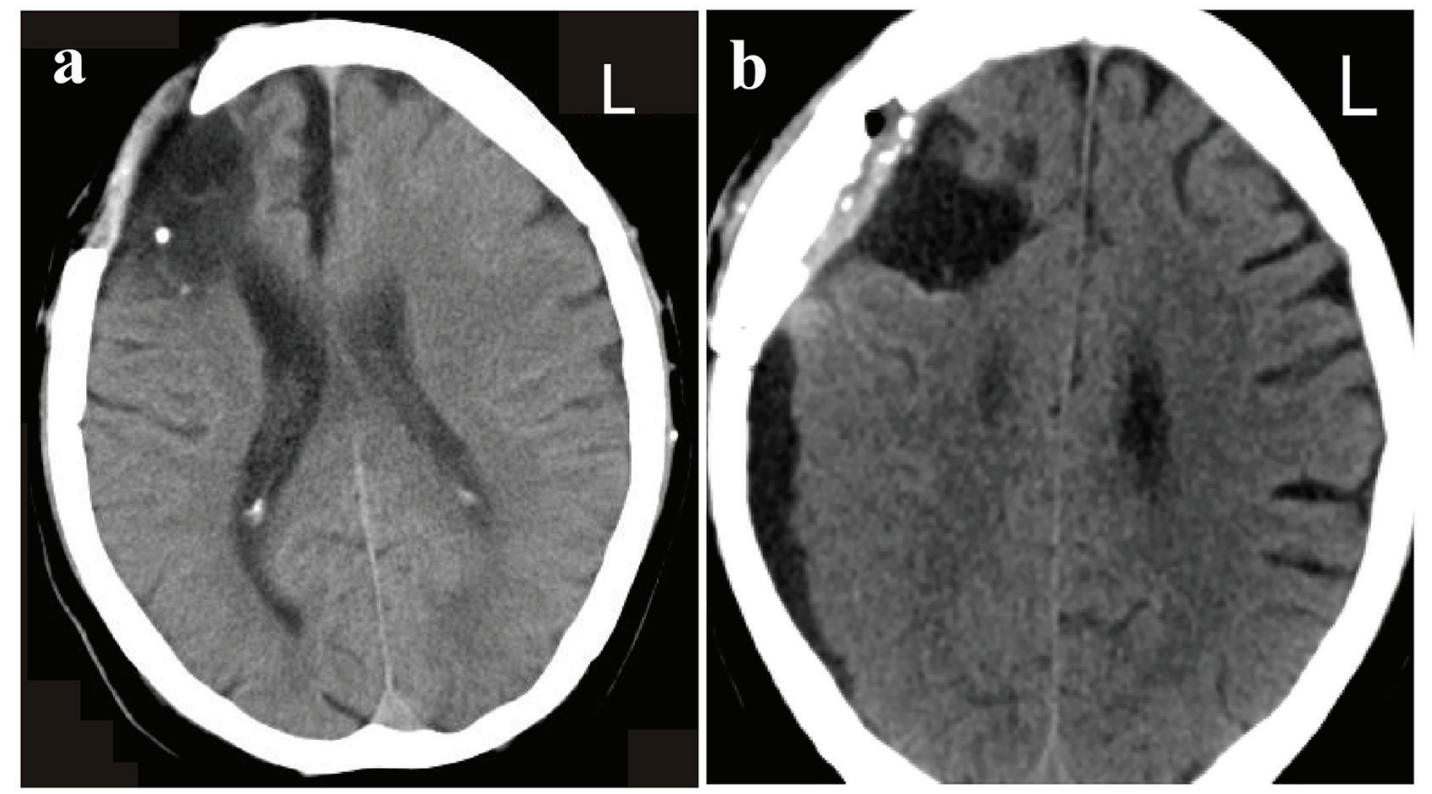

Figure 2. (a) CT brain scan before operation shows a large hypodense defect zone in the right frontal lobe with two metal fragments located in the defect zone. The right lateral ventricle is enlarged and drawn to the outward. (b) CT brain scan in the third postoperative day shows resection of pathological tissues and removal of metal fragments.

ported [12]. The occurrence of bilateral myoclonus following brain surgery and temporal lobe resection has been reported in only a few patients [6-8]. Bilateral non-epileptic myoclonus after resection of the frontal lobe, to our knowledge, has never been reported. Although polygraphic EEG-EMG was not performed, the lack of any correlated epileptiform discharges with the bilateral pattern of the myoclonic jerks in the postoperative EEG makes the diagnosis of epileptic myoclonus very unlikely. The motor neocortex is the most commonly observed myoclonus source, but origins from subcortical areas, brainstem, spinal, and peripheral nervous system also have been reported [13]. If surgical manipulation in our patient provoked seizure activity in deep brain structures, it is possible that epileptiform discharges might not be detected by scalp EEG. This is also very unlikely. Bilateral myoclonus in our patient could not be assumed as a drug-induced myoclonus as he had been treated for a long time with the same antiepileptic drugs before surgery without any side effects. There was also no evidence of any hypoxic damage, infection, and metabolic or toxic encephalopathies during or after the surgery.

It has been reported that the myoclonus can be induced by corticobasal neuronal dysfunction [14]. Any imbalance in motor cortex physiological activities may produce myoclonic twitching. Motor cortex hyperexcitability (such as lithium-induced myoclonus) or inhibition of neocortical excitability (like gabapentin- or pregabalin-induced myoclonus) could cause myoclonus [13]. Increased neuronal excitability has been suggested to enhance the potential of the lesioned brain for adaptive neuroplastic processes [15], change the cortical neuroplasticity [16], facilitate the flow of the motor neuron signals to the subcortical regions [17] and induce muscle jerking. This case report indicates that accurate diagnosis of this rare complication after frontal lobe resection ensures appropriate definition of surgical outcome in patients with intractable epilepsy.

\section{Conflict of Interest}

The authors declare that there are no conflicts of interest.

\section{Funding}

This study was supported by Iran National Science Foundation (INSF) to AG.

\section{References}

1. Lozsadi D. Myoclonus: a pragmatic approach. Pract Neurol. 2012;12(4):215-224.

2. Guerrini R, Takahashi T. Myoclonus and epilepsy. Handb Clin Neurol. 2013;111:667-679.

3. Kojovic M, Cordivari C, Bhatia K. Myoclonic disorders: a practical approach for diagnosis and treatment. Ther Adv Neurol Disord. 2011;4(1):47-62.

4. Kazemi H, Hashemi-Fesharaki S, Razaghi S, Najafi M, Kolivand PH, Kovac S, Gorji A. Intractable epilepsy and craniocerebral trauma: analysis of 163 patients with blunt and penetrating head injuries sustained in war. Injury. 2012;43(12):2132-2135.

5. Kerz T, Jantzen JP. [A myoclonic seizure during propofol-alfentanil anesthesia?]. Anaesthesist. 1992;41(7):426430 .

6. Wilkins DE, Hallett M, Erba G. Primary generalised epileptic myoclonus: a frequent manifestation of minipoly- 
myoclonus of central origin. J Neurol Neurosurg Psychiatry. 1985;48(6):506-516.

7. Diehl B, Wyllie E, Rothner AD, Bingaman W. Worsening seizures after surgery for focal epilepsy due to emergence of primary generalized epilepsy. Neurology. 1998;51(4):1178-1180.

8. Koutroumanidis M, Hennessy MJ, Chandler C. Postoperative myoclonus simulating seizure recurrence: a rare complication of anterior temporal lobectomy. Br J Neurosurg. 2001;15(2):174-177.

9. Rey-Dios R, Cohen-Gadol AA. Technical nuances for surgery of insular gliomas: lessons learned. Neurosurg Focus. 2013;34(2):E6.

10. Jumao-as A, Brenner RP. Myoclonic status epilepticus: a clinical and electroencephalographic study. Neurology. 1990;40(8):1199-1202.

11. Bowdle TA, Rooke GA. Postoperative myoclonus and rigidity after anesthesia with opioids. Anesth Analg. 1994;78(4):783-786.
12. Hachisuka K, Ogata H, Kohshi K. Post-operative paraplegia with spinal myoclonus possibly caused by epidural anaesthesia: case report. Paraplegia. 1991;29(2):131-136.

13. Caviness JN, Brown P. Myoclonus: current concepts and recent advances. Lancet Neurol. 2004;3(10):598-607.

14. Brunt ER, van Weerden TW, Pruim J, Lakke JW. Unique myoclonic pattern in corticobasal degeneration. Mov Disord. 1995;10(2):132-142.

15. Liuzzi G, Horniss V, Lechner P, Hoppe J, Heise K, Zimerman M, Gerloff C, et al. Development of movement-related intracortical inhibition in acute to chronic subcortical stroke. Neurology. 2014;82(3):198-205.

16. Popa T, Milani P, Richard A, Hubsch C, Brochard V, Tranchant C, Sadnicka A, et al. The neurophysiological features of myoclonus-dystonia and differentiation from other dystonias. JAMA Neurol. 2014;71(5):612-619.

17. Cantello R, Gianelli M, Civardi C, Mutani R. Focal subcortical reflex myoclonus. A clinical and neurophysiological study. Arch Neurol. 1997;54(2):187-196. 